\title{
Interleukin-6 Gene Promoter-572 C Allele may Play a Role in Rate of Disease Progression in Multiple Sclerosis
}

\author{
Jun Yan ${ }^{1, *}$, Jia Liu ${ }^{1}$, Clement Yihao Lin ${ }^{1}$, Australia and New Zealand Multiple Sclerosis \\ Genetics Consortium (ANZGene) ${ }^{\dagger}$, Peter A. Csurhes ${ }^{1,2}$, Michael P. Pender ${ }^{2}$, \\ Pamela A. McCombe ${ }^{1}$ and Judith M. Greer ${ }^{1, *}$
}

1 The University of Queensland, Centre for Clinical Research, Royal Brisbane \& Women's Hospital, 4029 Brisbane, Australia; E-Mails: jia.liu3@uqconnect.edu.au (J.L.); clement.lin@uqconnect.edu.au (C.Y.L.); p.csurhes@uq.edu.au (P.A.C.); pamela.mccombe@uq.edu.au (P.A.M.)

2 School of Medicine, The University of Queensland, and Department of Neurology, Royal Brisbane \& Women's Hospital, 4029 Brisbane, Australia; E-Mail: m.pender@uq.edu.au

$\dagger$ Members of the ANZGene Consortium are listed in the Acknowledgements section.

* Authors to whom correspondence should be addressed; E-Mails: j.yan@uq.edu.au (J.Y.); j.greer@uq.edu.au (J.M.G.); Tel.: +61-7-3346-6019 (J.Y.); Fax: +61-7-3346-5594 (J.Y. \& J.G.).

Received: 2 August 2012; in revised form: 20 September 2012 / Accepted: 27 September 2012 / Published: 22 October 2012

\begin{abstract}
Multiple sclerosis (MS) is an inflammatory demyelinating disease affecting the central nervous system. Although the exact pathogenesis of MS is unknown, it is generally considered to be an autoimmune disease, with numerous genetic and environmental factors determining disease susceptibility and severity. One important mediator of immune responses and inflammation is interleukin-6 (IL-6). Previously, elevated levels of IL-6 in mononuclear cells in blood and in brain tissue from MS patients have been reported. Various polymorphisms in the promoter region of the IL 6 gene have also been linked with IL-6 protein levels. In MS, several small studies have investigated whether two IL6 promoter polymorphisms ( $-597 \mathrm{G}>\mathrm{A}$ and $-174 \mathrm{G}>\mathrm{C}$ ) correlate with MS susceptibility, but with varying results. In the present study, we analyzed these polymorphisms, together with an additional polymorphism $(-572 \mathrm{G}>\mathrm{C})$ in 279 healthy controls and 509 patients with MS. We found no significant differences between MS patients and healthy controls for the different -597 or -174 IL6 promoter alleles or genotypes. There was a slight reduction in the percentage of individuals with MS who carried a $\mathrm{C}$ allele at position -572 , although
\end{abstract}


this was not significant after correction for multiple comparisons. Interestingly, however, the $-572 \mathrm{C}$ allele showed a significant correlation with the MS severity score, suggesting a possible role in disease progression.

Keywords: multiple sclerosis; Interleukin-6; polymorphism; allele; genotype; disease progression

\section{Introduction}

Multiple sclerosis (MS) is a chronic inflammatory demyelinating disease of the central nervous system (CNS) that affects more than 2.5 million people worldwide [1]. The majority of MS patients initially develop MS with a relapsing-remitting disease course (RR-MS) and about 50\% of those patients will subsequently develop secondary progressive disease (SP-MS). In 10\%-15\% of MS cases, however, disease is progressive from the outset and is known as primary progressive MS (PP-MS) [2]. RR-MS affects females more frequently than males (3:1), but in PP-MS, males and females are equally affected [3]. One of the hallmarks of MS is the unpredictability of the rate of progression of disease; some patients rapidly progress, whereas others may go many years without accumulation of significant disability.

The causes of MS are uncertain, and might not be identical in all individuals. It is believed that, in the majority of patients, the disease is maintained and driven by the development of autoimmune reactivity directed against myelin or neuronal components [4,5]; however, the exact precipitating events that allow the development of autoimmunity are not clear, and it is likely that both an underlying genetic susceptibility and exposure to one or more environmental triggers are required. Recent studies have identified numerous gene polymorphisms that appear to relate to MS susceptibility [5-7], although for many of these genes the effects are relatively small. Little is known, however, about genes that affect MS severity or the clinical course of MS.

Several studies have investigated whether two promoter region polymorphisms in the gene encoding interleukin 6 (IL-6), a pleiotropic cytokine that is an important mediator of many inflammatory processes, including induction of the acute phase response and differentiation of lymphocytes and monocytes, might enhance susceptibility to MS [8-11]. Increased levels of IL-6 have been detected in mononuclear cells in the blood and cerebrospinal fluid (CSF) [12-14] and in brain tissue of patients with MS [15]. Furthermore, studies from both human MS patients [13] and from mouse models of MS [16] suggest that IL-6 levels may correlate with disease severity. Because it is known that some of the IL6 promoter region polymorphisms can affect IL-6 levels, it is of interest to investigate the relationship between these polymorphisms, and MS susceptibility and severity.

The two IL6 polymorphisms that have thus far been investigated in MS are -597 G/A (rs 1800797) and $-174 \mathrm{G} / \mathrm{C}$ (rs1800795); these are in very strong linkage disequilibrium [17]. The $-174 \mathrm{C}$ allele can suppress IL-6 transcription [18], but less is known of the functional correlates of other IL6 promoter polymorphisms. In the MS studies, two groups reported no linkage between the -597 and -174 IL6 promoter polymorphisms and MS [8,9], whereas the other 2 studies did report significant correlations, but with different alleles/genotypes [10,11]. In all of these studies, the numbers of patients were fairly 
low, and few PP-MS patients were tested. We have not been able to ascertain whether rs 1800795 and rs1800797 were on the chips used in a large recent international MS GWAS [5], but have confirmed that they were not represented on the chips used in the ANZGene MS GWAS [6].

In kidney allograft survival studies, it has been found that the -597 and -174 IL6 promoter polymorphisms can operate cooperatively with another IL6 promoter polymorphism at -572 to influence graft survival [19], suggesting that it might be necessary to investigate the broader IL6 haplotype in order to determine if IL6 promoter polymorphisms are of functional relevance. The -572 G $>$ C polymorphism (rs 1800796) has not previously been studied in MS. The aim of the present study was therefore to investigate the IL6 promoter polymorphisms $-597,-572$ and -174 in a large group of MS patients and controls, and establish their relationship to MS susceptibility and progression.

\section{Results and Discussion}

\subsection{Allelic and Genotypic Frequency of the IL6 Promoter Region Polymorphisms}

Genomic DNA samples from patients with MS $(n=509)$ and healthy controls $(n=279)$ (see Table 1 for details of participants) were sequenced to determine the alleles and genotypes present in each individual (Table 2).

Table 1. Details of participants.

\begin{tabular}{ccccc}
\hline Group & $\boldsymbol{n}$ & \% Female & Age (Mean \pm SE) & $\begin{array}{c}\text { MSSS * } \\
\text { median (IQR) }\end{array}$ \\
\hline $\begin{array}{c}\text { Healthy controls } \\
\text { Multiple sclerosis }\end{array}$ & 279 & $63.1 \%$ & $42 \pm 1.0$ & not applicable \\
All & 509 & $68.4 \%$ & $52.8 \pm 0.5$ & $5.24(2.33-7.66)$ \\
RR-MS & 156 & $82.1 \%{ }^{\dagger}$ & $46.6 \pm 0.9$ & $1.73(0.49-3.42)$ \\
SP-MS & 172 & $69.2 \%$ & $53.4 \pm 0.8$ & $6.28(4.13-8.20)$ \\
PP-MS & 181 & $56.9 \%$ & $57.6 \pm 0.7$ & $6.90(4.82-8.49)$ \\
\hline
\end{tabular}

* The MS Severity Scores (MSSS) [20] were not normally distributed, and are therefore presented as the median and interquartile range (IQR). ${ }^{\dagger}$ The proportion of females in the RR-MS group was significantly different from that in the healthy control group $\left(p=3.5 \times 10^{-5}\right)$, the SP-MS group $(p=0.007)$ and the PP-MS group $\left(p=7 \times 10^{-7}\right)$.

There were no differences in the frequency of any of the IL6 promoter region polymorphisms between MS patients and controls, apart from a slight decrease in the frequency of the $-572 \mathrm{C}$ allele and CC genotype in patients with MS compared to healthy controls; these were not significant after correction for multiple comparisons. Interestingly, the decrease was not seen in patients with PP-MS. Our results are in striking contrast to those of both Shahbazi et al., who found an increased risk for MS associated with the G allele of -174 in an Iranian cohort [11], and of Mirowska-Guzel et al, who found increased risk of MS to be associated with the $\mathrm{C}$ allele of -174 , particularly with the CC genotype in a Polish cohort [10]. Whether these differences are due to differences in the ethnicity of the populations, or to some other factor, remains to be determined. A meta analysis of the -174 IL6 promoter region region genotype findings from the 4 previous studies in MS and the present study is shown in Figure 1. The numbers of samples in each of these studies were: Mirowska-Guzel et al. [10] 228 MS and 
193 controls; Shahbazi et al. [11] 345 MS and 426 controls; Mihailova et al. [9] 55 MS and 86 controls; Fedetz et al. [8] 131 MS and 157 controls; present study 509 MS and 279 controls.

Table 2. Results of allelic and genotypic tests for the IL6 promoter polymorphisms.

\begin{tabular}{|c|c|c|c|c|c|c|c|}
\hline \multirow{2}{*}{\multicolumn{2}{|c|}{$\begin{array}{c}\text { Allele/ } \\
\text { genotype }\end{array}$}} & \multirow{2}{*}{$\begin{array}{l}\text { Healthy } \\
\text { controls }\end{array}$} & \multicolumn{4}{|c|}{ Multiple sclerosis } & \multirow{2}{*}{$\begin{array}{c}P_{\text {uncorrected }} v s . \\
\text { healthy controls }\end{array}$} \\
\hline & & & All & RR-MS & SP-MS & PP-MS & \\
\hline \multirow[t]{5}{*}{-597} & $\mathrm{G}$ & $318^{(57.0 \%)}$ & $607^{(59.6 \%)}$ & $182^{(58.3 \%)}$ & $209^{(60.8 \%)}$ & $216^{(59.7 \%)}$ & \multirow[b]{2}{*}{ n.s. } \\
\hline & A & $240^{(43.0 \%)}$ & $411^{(40.4 \%)}$ & $130^{(41.7 \%)}$ & $135^{(39.2 \%)}$ & $146^{(40.3 \%)}$ & \\
\hline & GG & $94^{(33.7 \%)}$ & $181^{(35.6 \%)}$ & $54^{(34.6 \%)}$ & $64^{(37.2 \%)}$ & $63^{(34.8 \%)}$ & n.s. \\
\hline & GA & $130^{(46.6 \%)}$ & $245^{(48.1 \%)}$ & $74^{(47.4 \%)}$ & $81^{(47.1 \%)}$ & $90^{(49.7 \%)}$ & n.s. \\
\hline & AA & $55^{(19.7 \%)}$ & $83^{(16.3 \%)}$ & $28^{(17.9 \%)}$ & $27^{(15.7 \%)}$ & $28^{(15.5 \%)}$ & n.s. \\
\hline \multirow[t]{5}{*}{-572} & $\mathrm{G}$ & $517^{(92.7 \%)}$ & $968^{(95.1 \%)}$ & $301^{(96.5 \%)}$ & $328^{(95.3 \%)}$ & $339^{(93.6 \%)}$ & 0.047 (all MS); \\
\hline & $\mathrm{C}$ & $41^{(7.3 \%)}$ & $50^{(4.9 \%)}$ & $11^{(3.5 \%)}$ & $16^{(4.7 \%)}$ & $23^{(6.4 \%)}$ & 0.03 (RR-MS) \\
\hline & GG & $244^{(87.5 \%)}$ & $461^{(90.6 \%)}$ & $145^{(92.9 \%)}$ & $157^{(91.3 \%)}$ & $159^{(87.8 \%)}$ & n.s. \\
\hline & GC & $29^{(10.4 \%)}$ & $46^{(9.0 \%)}$ & $11^{(7.1 \%)}$ & $14^{(8.1 \%)}$ & $21^{(11.6 \%)}$ & n.s. \\
\hline & $\mathrm{CC}$ & $6^{(2.2 \%)}$ & $2^{(0.4 \%)}$ & $0^{(0 \%)}$ & $1^{(0.6 \%)}$ & $1^{(0.6 \%)}$ & 0.047 (all MS) \\
\hline \multirow[t]{5}{*}{-174} & $\mathrm{G}$ & $307^{(55.0 \%)}$ & $592^{(58.2 \%)}$ & $176^{(56.4 \%)}$ & $204^{(59.3 \%)}$ & $212^{(58.6 \%)}$ & \multirow{2}{*}{ n.s. } \\
\hline & $\mathrm{C}$ & $251^{(45.0 \%)}$ & $426^{(41.8 \%)}$ & $136^{(43.6 \%)}$ & $140^{(40.7 \%)}$ & $150^{(41.4 \%)}$ & \\
\hline & GG & $86^{(30.8 \%)}$ & $173^{(34.0 \%)}$ & $50^{(32.1 \%)}$ & $62^{(36.0 \%)}$ & $61^{(33.7 \%)}$ & n.s. \\
\hline & GC & $135^{(48.4 \%)}$ & $246^{(48.3 \%)}$ & $76^{(48.7 \%)}$ & $80^{(46.5 \%)}$ & $90^{(49.7 \%)}$ & n.s. \\
\hline & $\mathrm{CC}$ & $58^{(20.8 \%)}$ & $90^{(17.7 \%)}$ & $30^{(19.2 \%)}$ & $30^{(17.4 \%)}$ & $30^{(16.6 \%)}$ & n.s. \\
\hline
\end{tabular}

n.s.: not significant.

Figure 1. Meta analysis of the odds ratios in the five studies that have investigated -174 IL6 promoter region polymorphisms in MS. * This study inferred the -174 genotypes from the -597 genotype.

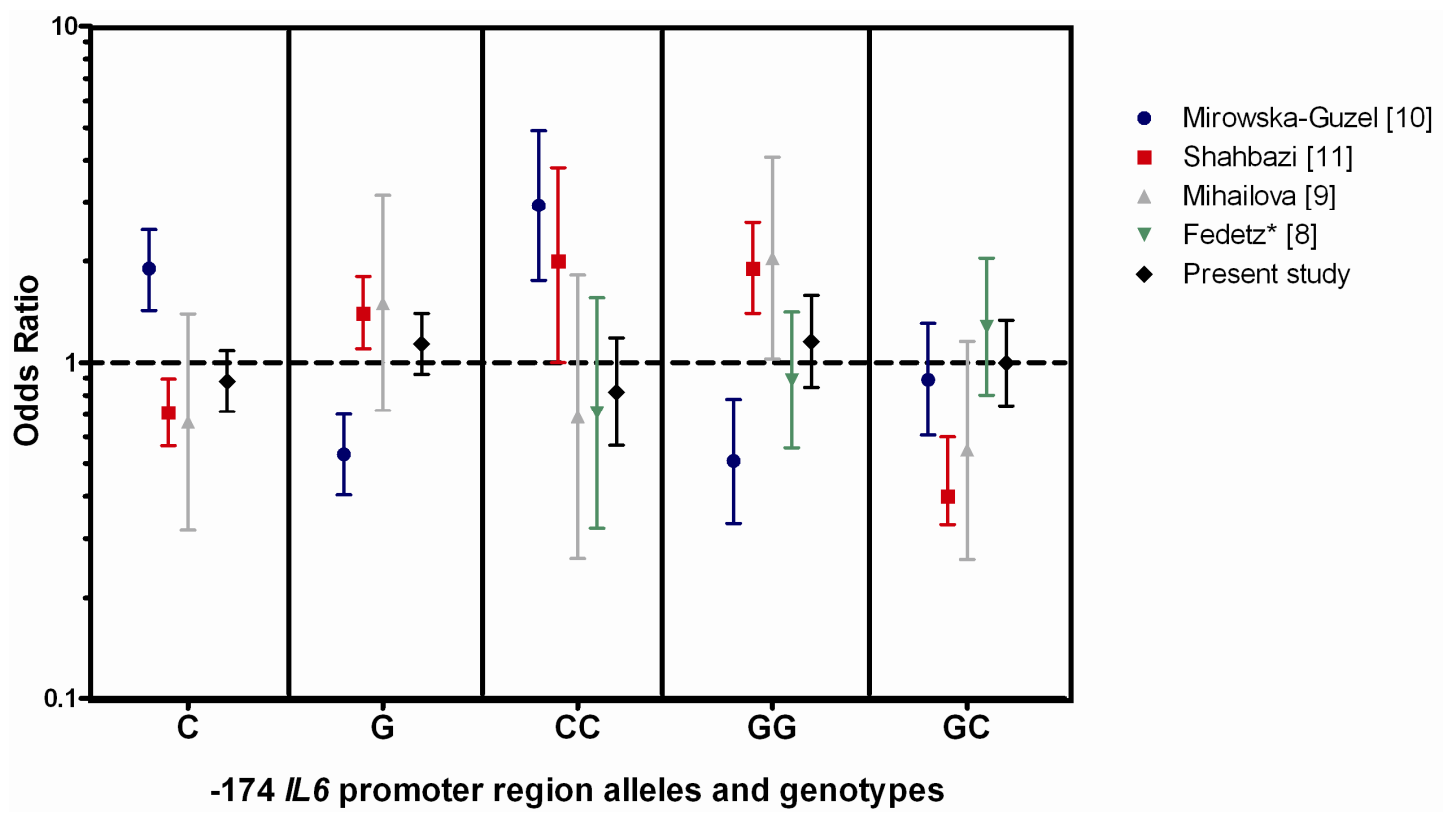




\section{2. -572 IL6 Promoter Region Genotypes, but not-172 Genotypes, Associate with MS Severity}

We next analyzed whether carriage of the different IL6 promoter region genotypes correlated with the severity of MS. The MS Severity Score (MSSS) [20] takes into account both the level of disease disability and the duration of disease, and is scored from 0 to 10 , with 10 being death from MS. Patients with the -572 GC genotype overall showed a significantly higher median MSSS than did those with the GG genotype $(p<0.05$ ) (Figure 2A). Only 2 of the 509 MS patients carried the CC genotype, and so no conclusions can be drawn regarding the effect of this on MS, although those two individuals did have a relatively high MSSS. In contrast, there was no difference in the median or IQR of the MSSS of patients with the different-174 genotypes (Figure 2B).

Figure 2. (A) Distribution of MS Severity Scores (MSSS) according to patient -572 genotype. Each bar on the graphs represents the median and interquartile range. The whiskers represent the 5th and 95th percentiles, and outliers are shown as black circles above and below the plots; (B) Distribution of MSSS in patients subdivided on basis of -174 genotype.
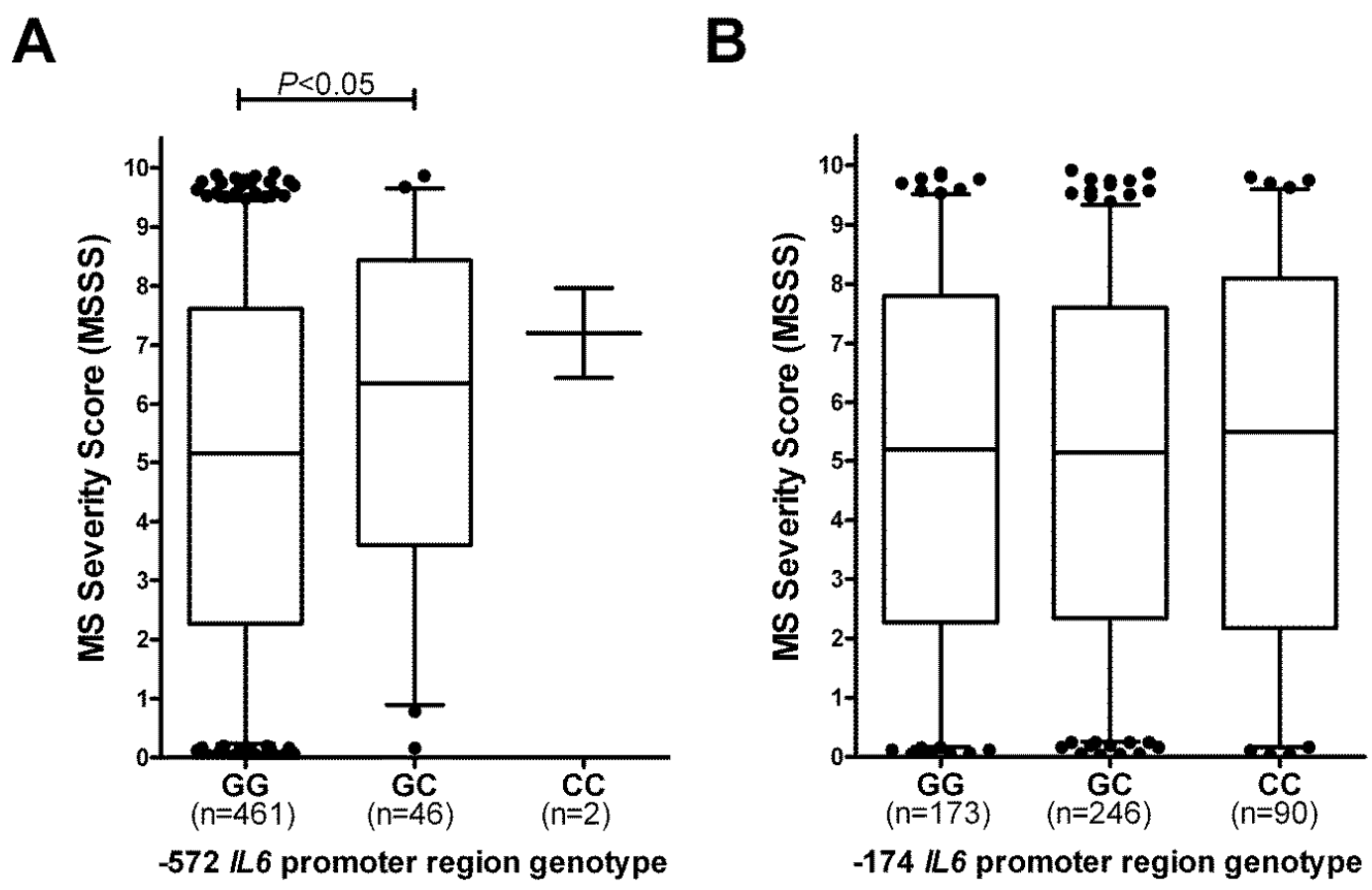

\subsubsection{Interaction of Gender with Genotype}

Because there were significant differences in the proportion of females with RR-MS compared to the other types of MS in our study, and because previous studies have identified the $-174 \mathrm{G}>\mathrm{C}$ polymorphism as a $17 \beta$-estradiol sensitive site in females with type 1 diabetes [21], we next analyzed whether carriage of the different IL6 promoter region genotypes differed according to the gender of the patients. For the -572 promoter polymorphism, as there was only 1 female and 1 male who had the CC genotype, GC and CC genotypes were considered together. Females with a GC/CC genotype showed a significantly ( $p=0.005$ ) elevated MSSS compared to females of GG genotype (Figure 3 ). For the other 
two IL6 promoter polymorphisms ( $-597 \mathrm{G}>\mathrm{A}$ and $-174 \mathrm{G}>\mathrm{C})$, there were no differences between males and females for the median MSSS scores (not shown).

Figure 3. MS Severity Scores in females and males, by -572 IL6 genotype.

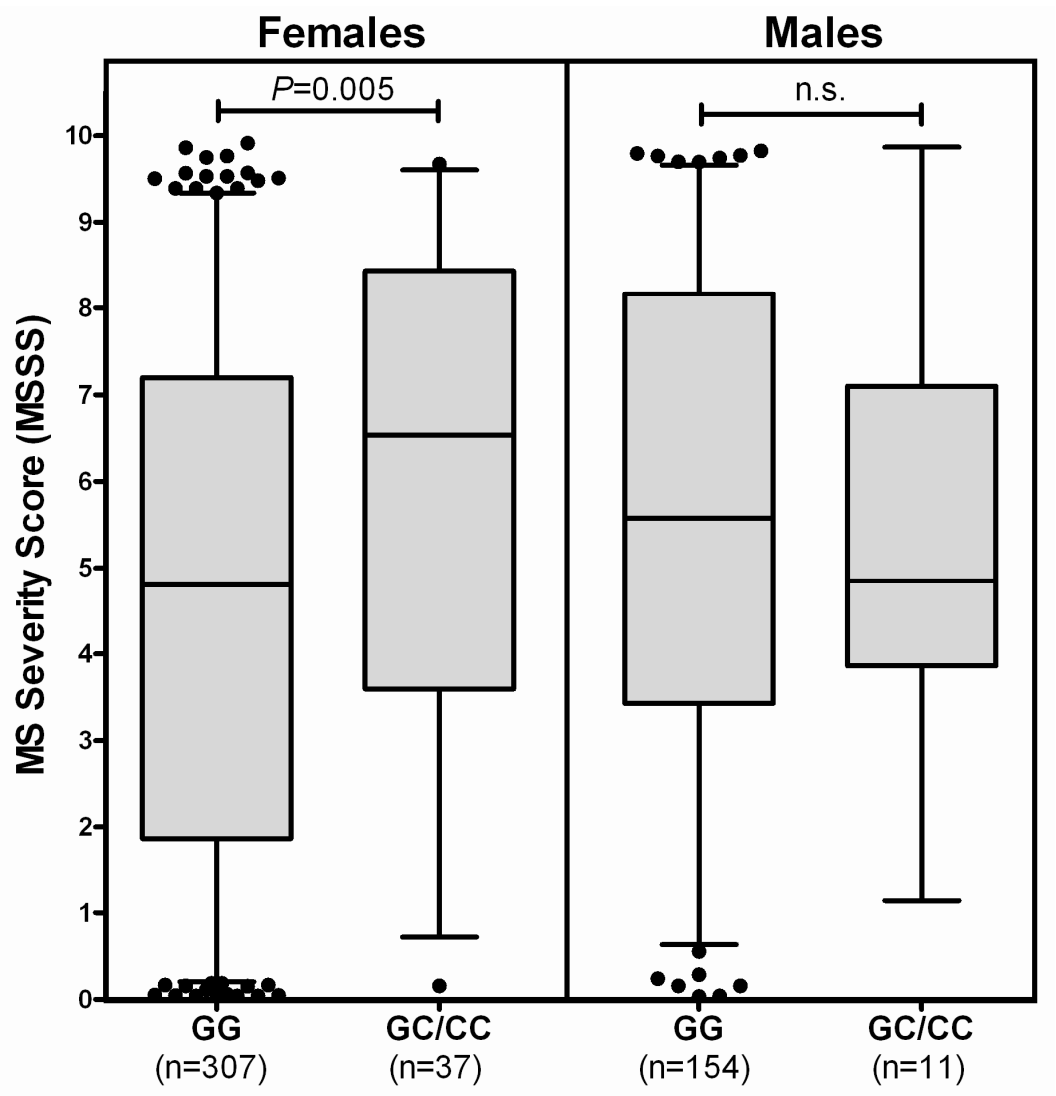

-572 IL6 promoter region genotype

Previously, it has been shown in type 1 diabetes that the -174 IL6 genotype is associated with the age of onset of disease in females, but not males [21]. Therefore, we looked at whether the genotype was associated with the age of onset of disease for the -572 and -174 genotypes. For the -572 genotypes, as there was only 1 female and 1 male who had the CC genotype, GC and CC genotypes were considered together. It has been established previously that females typically have a slightly earlier age of onset of MS than do males [22], and this was reflected in the analysis of the genotypes, where females typically developed MS 2-3 years earlier than males, particularly for age of onset between 25 and 50 years (Figure 4). This is most clearly seen when comparing the GG genotypes for -572 , where the line for the females (particularly for age of onset between 25 and 50) is to the left and higher than the male GG group. There was no association between genotype and age of onset of MS in females. There appeared to be a slight decrease in the time to disease onset in males who carried the $-572 \mathrm{GC}$ or CC genotypes (Figure 4A); however, there were only 11 males in this group, which precludes drawing any firm conclusions. For the -174 IL6 genotypes, there was no significant effect of the different genotypes on age of onset of MS (Figure 4B). 
Figure 4. Cumulative fraction of patients carrying different IL6 promoter genotypes vs. age of onset of MS. (A) Patients grouped according to -572 IL6 genotype; (B) Patients grouped according to -174 IL6 genotype.

A

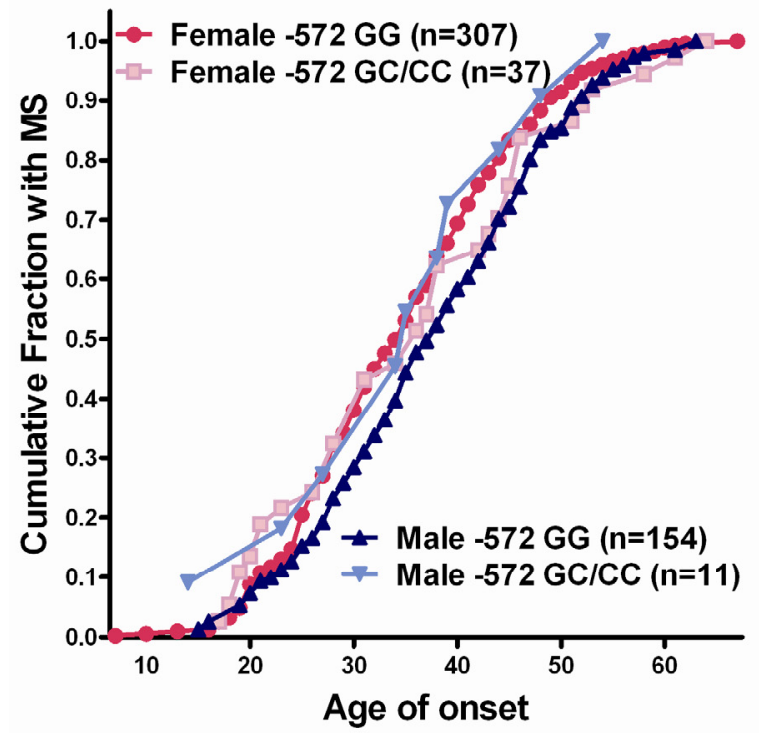

B

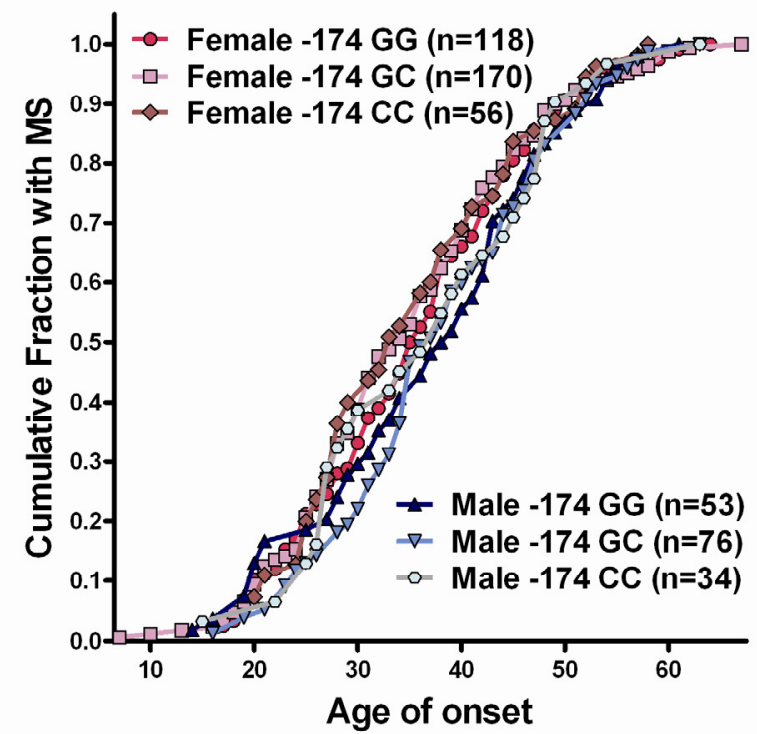

2.2.2. The -572 IL6 GC Genotype Association with MS Severity is Most Noticeable in Patients Who Initially Have RR-MS

The ratio of females to males with MS is significantly different in the group of patients who initially have RR-MS (i.e., the RR-MS + SP-MS groups), compared to patients who have PP-MS. Because we found that disease severity was elevated in females, but not males, of the $-572 \mathrm{GC} / \mathrm{CC}$ genotype (Figure 2), and because the allele frequency of the $-572 \mathrm{C}$ allele was somewhat reduced in MS compared to controls, particularly in the patients with RR-MS (Table 1), we therefore next investigated whether the MSSS differed in patients with different -572 genotypes who were subdivided on the basis of disease course. There was a significant difference between the MSSS of GG and GC/CC patients who initially had a RR course of disease, but not for those who had a primary progressive disease course ( $p=0.02$; Figure 5). We considered whether the differences between GG and GC carriers in the RR/SP group could be due to a higher proportion of patients with SP-MS in the GC group; however, this does not appear to be the case, as there was no significant difference in the proportion of SPMS patients in the GC group compared to the proportion of SP-MS patients in the GG group $(57.7 \%$ vs. $48.7 \% ; p=0.53)$. 
Figure 5. MS Severity Scores in patients who initially have a RR-MS course, compared to a PP-MS course, by -572 IL6 genotype.

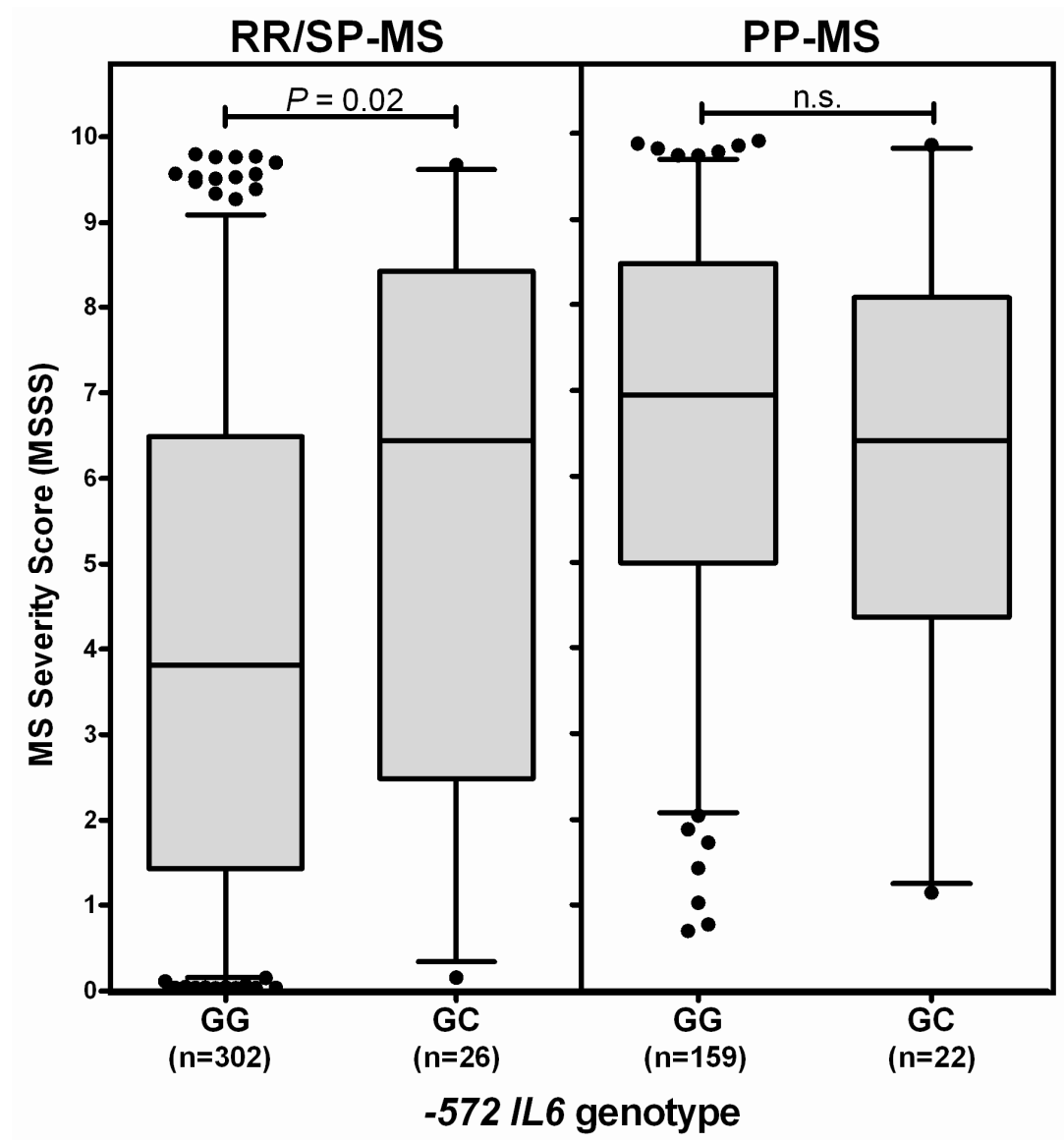

\subsubsection{Interaction of HLA Type with IL6 Promoter Region Genotype}

One of the recent publications that found significant differences in the -174 IL6 promoter region genotypes between patients and controls were intensified in the subgroup of patients who carry HLA-DRB1*1501 [11]. In Caucasian populations, DRB1*1501 occurs approximately twice as frequently in people with MS than in healthy individuals. We therefore analyzed whether carriage of DRB1*1501 by MS patients was associated with the frequency of the different genotypes or with the MSSS in patients with different genotypes. Four hundred and ninety-one MS patients ( $96.5 \%$ of total) were typed to determine whether or not they carried HLA-DRB $1 * 1501$. Fifty-seven percent of all MS patients were positive for DRB1*1501. The percentage of DRB1*1501 positive MS patients was only marginally different between RR/SP-MS (57.6\%) and PP-MS (55.9\%). The ratio of DRB1*1501 positive to DRB1*1501 negative MS patients did not change significantly, irrespective of the IL6 alleles or genotypes of the patients (data not shown). Similarly, when the MSSS was compared between DRB1*1501 positive and negative individuals, stratified according to the -572 or -174 IL6 promoter region genotypes, there was no effect attributable to the HLA type (not shown). Therefore, in this sample, we were unable to confirm the previous results of an effect of DRB1*1501 on the IL6 genotype. 


\subsection{Haplotypes of the IL6 Promoter Region Polymorphisms}

One of the limitations of the method employed for DNA sequencing in this paper is that it is not possible to accurately assign haplotypes for heterozygous genotypes. However, a substantial proportion of the MS (45.4\%) and healthy control (44.4\%) populations are homozygous across each genotype, and therefore their haplotypes could be correctly assigned. We have therefore analyzed the frequency of carriage of these homozygous haplotypes in healthy controls and patients with MS (Table 3). There were no significant differences between healthy controls and MS patients for these haplotypes. Futhermore, there were no significant differences in the MSSS of patients of these different haplotypes, or between patients who were homozygous or heterozygous (Figure 6).

Table 3. Frequency of IL6 promoter region haplotypes, for those individuals who had identical haplotypes on each DNA strand.

\begin{tabular}{|c|c|c|c|c|c|c|}
\hline \multirow{2}{*}{$\begin{array}{c}\text { Homozygous } \\
\text { haplotype } \\
(-597,-572,-174)\end{array}$} & \multirow{2}{*}{$\begin{array}{l}\text { Healthy } \\
\text { controls } \\
(n=124)\end{array}$} & \multicolumn{4}{|c|}{ Multiple sclerosis } & \multirow{2}{*}{$\begin{array}{c}P_{\text {uncorrected }} v s \\
\text { healthy } \\
\text { controls }\end{array}$} \\
\hline & & $\begin{array}{c}\text { All } \\
(n=231)\end{array}$ & $\begin{array}{l}\text { RR-MS } \\
(n=72)\end{array}$ & $\begin{array}{l}\text { SP-MS } \\
(n=84)\end{array}$ & $\begin{array}{l}\text { PP-MS } \\
(n=75)\end{array}$ & \\
\hline GGG & $85^{(68.5 \%)}$ & $146^{(63.2 \%)}$ & $45^{(62.5 \%)}$ & $53^{(63.1 \%)}$ & $48^{(64.0 \%)}$ & n.s. \\
\hline ACG & $39^{(31.5 \%)}$ & $81^{(35.1 \%)}$ & $27^{(37.5 \%)}$ & $29^{(34.5 \%)}$ & $25^{(33.3 \%)}$ & n.s. \\
\hline Other & $0^{(0 \%)}$ & $4^{(1.7 \%)}$ & $0^{(0 \%)}$ & $2^{(2.4 \%)}$ & $2^{(2.7 \%)}$ & n.s. \\
\hline
\end{tabular}

Figure 6. MSSS of patients with different IL6 promoter region haplotypes. There were no significant differences between patients with different haplotypes.

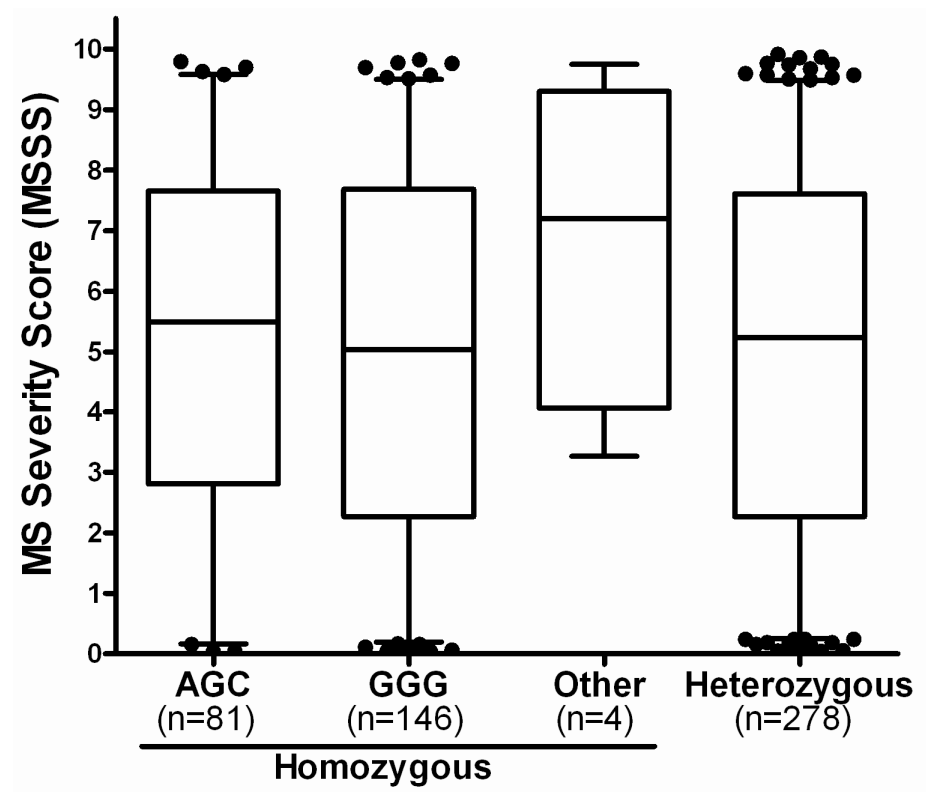

\section{Experimental Section}

\subsection{Patients and Controls}

Our study was reviewed and approved by the Royal Brisbane and Women's Hospital Health Service District Office of the Human Research Ethics Committee and by the Medical Research Ethics Committee, The University of Queensland, Brisbane, Australia. Blood or saliva was collected, after 
informed consent was obtained, from 279 healthy controls and 509 patients with MS. The age, sex and, for the MS patients, clinical course of disease and MSSS are shown in Table 1. The MSSS is an algorithm which relates scores on the Kurtzke Expanded Disability Status Scale (EDSS) to the distribution of disability in patients with comparable disease durations [20]. The MSSS is scored from 0.01 (least severe) to 9.99 (most severe). The MS patients were all recruited by the Australia and New Zealand Multiple Sclerosis Genetics Consortium (ANZgene) and met the 2005 revised McDonald criteria for MS [23]. Healthy controls were recruited from Royal Brisbane and Women's Hospital or University of Queensland staff.

\subsection{Genomic DNA Isolation and Sequencing}

Genomic DNA was extracted from healthy control blood samples using a Qiagen blood DNA extraction kit (Qiagen, Australia). Genomic DNA samples from either blood or saliva of MS patients were provided by ANZgene consortium and were prepared as previously described [6]. The DNA concentration was determined using a NanoDrop ${ }^{\mathrm{TM}} 1000$ Spectrophotometer (Thermo Scientific, Waltham, MA, USA). DNA sequencing was done at the Australian Genomic Research Facility, Brisbane, Queensland, Australia or at the Beijing Genomic Institute, ShenZhen, China. PCR primers used for sequencing IL-6 -597 G>A and $-572 \quad \mathrm{G}>\mathrm{C}$ polymorphisms were 5'-TGAGGCTAGCGCTAAGAAGC-3' (forward) and 5'-CTGGGAGGATTCCCAAGG-3' (reverse). For sequencing the $-174 \mathrm{G}>\mathrm{C}$ polymorphism the primers were $5^{\prime}$-AGACATGCCAAAGTGCTGAGT-3' (forward) and 5'-GCTCCTGGAGGGGAGATAGA-3' (reverse).

\subsection{Data Analysis}

Comparisons of allele and genotype frequencies between healthy controls and MS patients (and subgroups of MS) was made by $\chi^{2}$ analysis. For comparison of MSSS values in patients of different genotypes, all data were first checked to determine if they were normally distributed. The MSSS values were not normally distributed, and therefore were analyzed using the non-parametric Mann-Whitney test for comparison of 2 populations or the Kruskal-Wallis analysis of variance method for comparison of 3 or more populations.

\section{Conclusions}

The -572 IL6 promoter region C allele appears to correlate with the MSSS, which combines measures of the disease severity and disease duration, even though IL6 promoter region polymorphisms do not appear to associate strongly with susceptibility to MS. A caveat to this is that because the sample size is still relatively small, and the frequency of the $\mathrm{C}$ allele is relatively low, this observation will need to be replicated to confirm whether IL6 promoter region polymorphisms are indeed a predictor of MS severity.

The correlation between the -572 IL6 promoter region C allele and MS severity score is particularly noticeable in female patients, when patients are divided according to gender, which is of interest, as estrogen is a well-known modulator of IL6 gene expression. 
The clinical subtype of MS also affected the correlation between MSSS and -572 IL6 genotype. This could be because the percentage of females in the RR-MS group was significantly greater than the percentage of females in the PP-MS group, or for other reasons not yet elucidated.

It is not known whether the -572 IL6 promoter region polymorphism influences IL-6 levels; further in vitro functional assays will be required to prove whether the $-572 \mathrm{C}$ allele alone, or haplotypes containing this allele, regulate the expression of IL-6.

We were unable to reproduce previous studies suggesting that polymorphisms at -174 of the IL6 promoter region correlate with susceptibility to MS [10,11]. The reasons for this could be due to differences in the ethnicity of patients in those studies and the current study, the sample size used in the studies, or to other factors as yet undetermined.

\section{Acknowledgments}

Financial support for this project was provided by MS Research Australia and the Royal Brisbane and Women's Hospital Research Foundation.

Members of the ANZGene consortium are:

Rodney J. Scott ${ }^{1,2,3}$, Jeannette Lechner-Scott ${ }^{1,2}$, Matthew A. Brown ${ }^{4,5}$, David R. Booth ${ }^{6}$, Graeme J. Stewart ${ }^{6}$, Simon Broadley ${ }^{7,8}$, Deborah Mason ${ }^{9}$, Lyn Griffiths ${ }^{10}$, Pablo Moscato ${ }^{3,11}$, Mark Slee ${ }^{11}$, Bruce Taylor ${ }^{12}$, James Wiley ${ }^{13}$, Judith Field ${ }^{13,14}$, Helmut Butzkueven 13,15,16, Trevor J. Kilpatrick ${ }^{13,17}$.

${ }^{1}$ John Hunter Hospital, Hunter New England Health Service, Newcastle, NSW 2310, Australia.

${ }^{2}$ Hunter Medical Research Institute, Newcastle, NSW 2308, Australia.

${ }^{3}$ Centre for Bioinformatics, Biomarker Discovery and Information-based Medicine, University of Newcastle, NSW 2308, Australia.

4 The University of Queensland, Diamantina Institute of Cancer, Immunology and Metabolic Medicine, Princess Alexandra Hospital, Brisbane, QLD 4102, Australia.

${ }^{5}$ Botnar Research Centre, Nuffield Department of Orthopaedic Surgery, University of Oxford, Oxford OX3 7BN, UK.

${ }^{6}$ The Westmead Millenium Institute, Westmead, NSW 2145, Australia.

${ }^{7}$ School of Medicine, Griffith University, QLD, Australia 4222.

${ }^{8}$ Department of Neurology, Gold Coast Hospital, QLD 4215, Australia.

${ }^{9}$ Canterbury District Health Board, Christchurch, New Zealand.

${ }^{10}$ Genomics Research Centre, Griffith University, QLD 4222, Australia.

11 School of Medicine, Department of Neurology, Flinders University of South Australia, Bedford Park, Adelaide, SA 5042, Australia.

${ }^{12}$ Menzies Research Institute, University of Tasmania, Hobart, Tasmania 7001, Australia.

${ }^{13}$ Florey Neuroscience Institutes, University of Melbourne, Melbourne, VIC 3010, Australia.

${ }^{14}$ Centre for Neuroscience, University of Melbourne, Melbourne VIC 3010, Australia.

${ }^{15}$ Department of Medicine, University of Melbourne, Melbourne, VIC 3010, Australia.

${ }^{16}$ Department of Neurology, Box Hill Hospital, VIC 3128, Australia.

${ }^{17}$ Royal Melbourne Hospital, Parkville, VIC 3050, Australia. 


\section{References}

1. Pender, M.P.; Greer, J.M. Immunology of multiple sclerosis. Curr. Allergy Asthma Rep. 2007, 7, 285-292.

2. Montalban, X. Primary progressive multiple sclerosis. Curr. Opin. Neurol. 2005, 18, 261-266.

3. Ebers, G.C. Natural history of primary progressive multiple sclerosis. Mult. Scler. 2004, 10, S8-S15.

4. McFarland, H.F.; Martin, R. Multiple sclerosis: A complicated picture of autoimmunity. Nat. Immunol. 2007, 8, 913-919.

5. The International Multiple Sclerosis Genetics Consortium. The Wellcome Trust Case Control Consortium Genetic risk and a primary role for cell-mediated immune mechanisms in multiple sclerosis. Nature 2011, 476, 214-219.

6. Bahlo, M.; Booth, D.R.; Broadley, S.A.; Brown, M.A.; Foote, S.J.; Griffiths, L.R.; Kipatrick, T.J.; Lechner-Scott, J.; Moscato, P.; Perreau, V.M.; et al. Genome-wide association study identifies new multiple sclerosis susceptibility loci on chromosomes 12 and 20. Nat. Genet. 2009, 41, 824-828.

7. Patsopoulos, N.A.; Bayer Pharma, M.S. Genetics Working Group; Steering Committees of Studies Evaluating IFN $\beta-1 b$ and a CCR1-Antagonist; ANZgene Consortium; GeneMSA; International Multiple Sclerosis Genetics Consortium; Esposito, F.; Reischl, J.; Lehr, S.; Bauer, D.; et al. Genome-wide meta-analysis identifies novel multiple sclerosis susceptibility loci. Ann. Neurol. 2011, 70, 897-912.

8. Fedetz, M.; Matesanz, F.; Pascual, M.; Martin, J.; Fernandez, O.; Guerrero, M.; Alcina, A. The -174/-597 promoter polymorphisms in the interleukin-6 gene are not associated with susceptibility to multiple sclerosis. J. Neurol. Sci. 2001, 190, 69-72.

9. Mihailova, S.; Ivanova, M.; Mihaylova, A.; Quin, L.; Mikova, O.; Naumova, E. Pro- and anti-inflammatory cytokine gene polymorphism profiles in Bulgarian multiple sclerosis patients. J. Neuroimmunol. 2005, 168, 138-143.

10. Mirowska-Guzel, D.; Gromadzka, G.; Mach, A.; Czlonkowski, A.; Czlonkowska, A. Association of IL1A, IL1B, ILRN, IL6, IL10 and TNF-alpha polymorphisms with risk and clinical course of multiple sclerosis in a Polish population. J. Neuroimmunol. 2011, 236, 87-92.

11. Shahbazi, M.; Ebadi, H.; Fathi, D.; Roshandel, D.; Mohamadhosseni, M.; Tahmasebi, A.; Shahbazi, S.; Zamani, M.; Rashidbaghan, A. HLA-DRB1*1501 intensifies the impact of IL-6 promoter polymorphism on the susceptibility to multiple sclerosis in an Iranian population. Mult. Scler. 2010, 16, 1173-1177.

12. Maimone, D.; Gregory, S.; Arnason, B.G.; Reder, A.T. Cytokine levels in the cerebrospinal fluid and serum of patients with multiple sclerosis. J. Neuroimmunol. 1991, 32, 67-74.

13. Stelmasiak, Z.; Koziol-Montewka, M.; Dobosz, B.; Rejdak, K.; Bartosik-Psujek, H.; Mitosek-Szewczyk, K.; Belniak-Legiec, E. Interleukin-6 concentration in serum and cerebrospinal fluid in multiple sclerosis patients. Med. Sci. Monit. 2000, 6, 1104-1108.

14. Ireland, S.J.; Blazek, M.; Harp, C.T.; Greenberg, B.; Frohman, E.M.; Davis, L.S.; Monson, N.L. Antibody-independent B cell effector functions in relapsing remitting multiple sclerosis: Clues to increased inflammatory and reduced regulatory B cell capacity. Autoimmunity 2012, 45, 400-414. 
15. Maimone, D.; Guazzi, G.C.; Annunziata, P. IL-6 detection in multiple sclerosis brain. J. Neurol. Sci. 1997, 146, 59-65.

16. Eugster, H.P.; Frei, K.; Kopf, M.; Lassmann, H.; Fontana, A. IL-6-deficient mice resist myelin oligodendrocyte glycoprotein-induced autoimmune encephalomyelitis. Eur. J. Immunol. 1998, 28, 2178-2187.

17. Fife, M.S.; Ogilvie, E.M.; Kelberman, D.; Samuel, J.; Gutierrez, A.; Humphries, S.E.; Woo, P. Novel IL-6 haplotypes and disease association. Genes Immun. 2005, 6, 367-370.

18. Fishman, D.; Faulds, G.; Jeffery, R.; Mohamed-Ali, V.; Yudkin, J.S.; Humphries, S.; Woo, P. The effect of novel polymorphisms in the interleukin-6 (IL-6) gene on IL-6 transcription and plasma IL-6 levels, and an association with systemic-onset juvenile chronic arthritis. J. Clin. Invest. 1998, 102, 1369-1376.

19. Muller-Steinhardt, M.; Fricke, L.; Muller, B.; Ebel, B.; Kirchner, H.; Hartel, C. Cooperative influence of the interleukin-6 promoter polymorphisms $-597,-572$ and -174 on long-term kidney allograft survival. Am. J. Transpl. 2004, 4, 402-406.

20. Roxburgh, R.H.; Seaman, S.R.; Masterman, T.; Hensiek, A.E.; Sawcer, S.J.; Vukusic, S.; Achiti, I.; Confavreux, C.; Coustans, M.; le Page, E.; et al. Multiple sclerosis severity score: Using disability and disease duration to rate disease severity. Neurology 2005, 64, 1144-1151.

21. Kristiansen, O.P.; Nolsøe, R.L.; Larsen, L.; Gjesing, A.M.P.; Johannesen, J.; Larsen, Z.M.; Lykkesfeldt, A.E.; Karlsen, A.E.; Pociot, F.; Mandrup-Poulsen, T.; DIEGG; DSGD. Association of a functional $17 \beta$-estradiol sensitive $I L 6-174 \mathrm{G} / \mathrm{C}$ promoter polymorphism with early-onset type 1 diabetes in females. Hum. Mol. Genet. 2003, 12, 1101-1110.

22. Sadovnick, A.D. European Charcot Foundation Lecture: The natural history of multiple sclerosis and gender. J. Neurol. Sci. 2009, 286, 1-5.

23. Polman, C.H.; Reingold, S.C.; Edan, G.; Filippi, M.; Hartung, H.P.; Kappos, L.; Lublin, F.D.; Metz, L.M.; McFarland, H.F.; O’Connor, P.W.; et al. Diagnostic criteria for multiple sclerosis: 2005 revisions to the "McDonald Criteria". Ann. Neurol. 2005, 58, 840-846.

(C) 2012 by the authors; licensee MDPI, Basel, Switzerland. This article is an open access article distributed under the terms and conditions of the Creative Commons Attribution license (http://creativecommons.org/licenses/by/3.0/). 